\title{
Importance of Risk Factors for Febrile Neutropenia Among Patients Receiving Chemotherapy Regimens Not Classified as High-Risk in Guidelines for Myeloid Growth Factor Use
}

\author{
Derek Weycker, $\mathrm{PhD}^{\mathrm{a}}$; Xiaoyan Li, $\mathrm{PhD}^{\mathrm{b}}$; Rich Barron, $\mathrm{MS}^{\mathrm{b}}$; Hongsheng Wu, PhD; ; P.K. Morrow, $\mathrm{MD}^{\mathrm{b}}$; \\ Hairong $\mathrm{Xu}, \mathrm{MD}, \mathrm{PhD}^{\mathrm{b}}$; Maureen Reiner, $\mathrm{MS}^{\mathrm{b}}$; Jacob Garcia, $\mathrm{MD}^{\mathrm{b}}$; Shivani K. Mhatre, MS ; and \\ Gary H. Lyman, MD, MPH ${ }^{d}$
}

\begin{abstract}
Background: Clinical practice guidelines recommend prophylaxis in patients with cancer receiving a colony-stimulating factor (CSF) when the risk of febrile neutropenia (FN) is high (>20\%). For patients receiving chemotherapy regimens not documented as high-risk, the decision regarding CSF prophylaxis use can be challenging, because some patients may be at high risk based on a combination of the regimen and individual risk factors. Methods: A retrospective cohort design and US private health care claims data were used. Study subjects received chemotherapy regimens classified as "low" or "intermediate," or unclassified, in terms of FN risk, and were stratified by cancer and regimen. For each subject, the first chemotherapy course, and each cycle and FN episode within the course, were identified. FN incidence proportions were estimated by the presence and number of risk factors and chronic comorbidities. Results: Across the 17 tumor/regimen combinations considered ( $n=160,304$ in total), $74 \%$ to $98 \%$ of patients had 1 or more risk factor for FN and $41 \%$ to $89 \%$ had 2 or more. Among patients with 1 or more risk factor, $\mathrm{FN}$ incidence ranged from $7.2 \%$ to $29.0 \%$ across regimens, and the relative risk of FN (vs those without risk factors) ranged from $1.1(95 \% \mathrm{Cl}, 0.8-1.3)$ to $2.2(95 \% \mathrm{Cl}, 1.5-3.0)$. FN incidence increased in a graded and monotonic fashion with the number of risk factors and comorbidities. Conclusions: In this retrospective evaluation of patients with cancer receiving chemotherapy regimens not classified as high-risk for FN in US clinical practice, most patients had 1 or more FN risk factor and many had 2 or more. FN incidence was found to be elevated in these patients, especially those with multiple risk factors. (J Natl Compr Canc Netw 2015;13:979-986)
\end{abstract}

\section{Background}

Neutropenia is a common side effect of myelosuppressive chemotherapy that increases the risk of infection, which is typically signaled by fever. When neutropenic patients develop fever (ie, febrile neutropenia [FN]), the likelihood of infection and serious consequences often necessitates

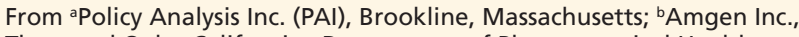
Thousand Oaks, California; 'Department of Pharmaceutical Health Outcomes and Policy, College of Pharmacy, Texas Medical Center, University of Houston, Houston, Texas; and dHutchinson Institute for Cancer Outcomes Research, Fred Hutchinson Cancer Research Center, University of Washington, Seattle, Washington.

Submitted December 5, 2014; accepted for publication May 21, 2015. The following authors have disclosed the following relationships: Dr. Weycker receives study support from Amgen; Dr. Li, Mr. Barron, Dr. Morrow, Dr. Xu, Ms. Reiner, and Dr. Garcia hold stock from and are employees of Amgen. Drs. Wu and Ms. Mhatre have disclosed that they have no financial interests, arrangements, affiliations, or commercial interests with the manufacturers of any products discussed in this article or their competitors. Dr. Lyman has disclosed that he is a PI for an institutional research grant from Amgen.
}

hospitalization. ${ }^{1} \mathrm{FN}$ can lead to chemotherapy dose delays, dose reductions, and/or discontinuation, interfering with the delivery of treatment and possibly adversely affecting patient outcomes. ${ }^{1-5}$

For adult patients receiving chemotherapy for solid tumors or nonmyeloid malignancies, NCCN Clinical Practice Guidelines in Oncology (NCCN Guidelines)
Funding for this research was provided by Amgen Inc. to Policy Analysis Inc. (PAI).

Author contributions: Study conception and supervision: D.W. and X.L.; Development of design: D.W., X.L., R.B., H.W., P.K.M., H.X., M.R., J.G., S.K.M., and G.H.L.; Conduct of analyses: D.W. and H.W.; Interpretation of results: D.W., X.L., R.B., H.W., P.K.M., H.X., M.R., J.G., S.K.M., and G.H.L.; Preparation of manuscript: D.W. and X.L.; Review of manuscript: D.W., X.L., R.B., H.W., P.K.M., H.X., M.R., J.G., S.K.M., and G.H.L. The study sponsor reviewed the study research plan and study manuscript. Data management, processing, and analyses were conducted by PAI, and all final analytic decisions were made by study investigators.

Correspondence: Derek Weycker, PhD, Policy Analysis Inc. (PAI), Four Davis Court, Brookline, MA 02445. E-mail: dweycker@pai2.com 
Weycker et al

for Myeloid Growth Factors $^{6}$ recommend prophylaxis against $\mathrm{FN}$ with a colony-stimulating factor (CSF) when risk, based on the chemotherapy regimen and patient risk factors, is "high" (>20\%) (to view the most recent version of these guidelines, visit NCCN.org). For patients whose risk is high based on the chemotherapy regimen alone, the decision to administer CSF prophylaxis is relatively straightforward. For patients receiving a regimen classified as "intermediate" (FN risk, $\geq 10 \%$ to $\leq 20 \%$ ), or even "low" (FN risk, $<10 \%$ ), however, careful consideration must be given to patient risk factors when calculating overall $\mathrm{FN}$ risk and deciding whether to administer CSF prophylaxis. ${ }^{1,5-14}$ Available evidence suggests that these patients are not rare; it has been estimated that approximately $50 \%$ of all adults receiving low- or intermediaterisk regimens have an $\mathrm{FN}$ risk greater than 20\% when taking into account their (ie, patient) risk factors. ${ }^{1,15-20}$ Available evidence also suggests that it is important to consider not only whether risk factors are present but also the number and type of risk factors present. ${ }^{11,14}$

Because of the many exclusion criteria used in clinical trials, patients in real-world clinical practice are likely to differ from trial subjects in significant ways. In addition, other factors, such as use of supportive care and chemotherapy dose delays and dose reductions during the treatment course, may lead to variation in FN risk profiles and events seen in the clinical trial and clinical practice settings. Understanding these risks in routine clinical practice, especially among patients receiving lowand intermediate-risk regimens, is thus important for treatment decision-making at the patient level and policy decision-making at the population level.

One source of data on the real-world complications of patients undergoing cancer chemotherapy is health care claims (or administrative) databases. Although lacking clinical richness, these databases provide access to the health care experience of millions of persons over a multiyear period, and thus contain information on large numbers of patients with specific cancer diagnoses who are treated with specific chemotherapy regimens. $^{21-25}$ We thus undertook one of the first comprehensive evaluations to estimate the prevalence of risk factors for $\mathrm{FN}$ among patients with cancer receiving chemotherapy regimens not classified as high-risk for FN in US clinical practice, and the risk of FN based on the presence and number of risk factors. The cancers and myelosuppressive chemotherapy regimens considered in the analyses described herein represent those commonly found in US clinical practice and for which the occurrence of $\mathrm{FN}$ is frequent, including breast cancer, colorectal cancer, lung cancer, and non-Hodgkin's lymphoma (NHL). ${ }^{3,6-10}$

\section{Methods}

\section{Study Design and Data Source}

A retrospective cohort design and data from 2 large integrated US health care claims repositories were used. The 2 repositories - the Truven Health Analytics MarketScan Commercial Claims and Encounters and Medicare Supplemental and Coordination of Benefits databases, and the IMS LifeLink PharMetrics Health Plan Claims database-comprise medical and outpatient pharmacy claims from a large number of private US health plans. Data for the current study spanned January 1, 2003, through December 31, 2012. The study databases were deidentified, and their use for health services research is fully compliant with the HIPAA Privacy Rule and federal guidance on Public Welfare and the Protection of Human Subjects. ${ }^{26} \mathrm{~A}$ detailed description of the data sources is provided in supplemental eAppendix 1 (available with this article at JNCCN.org).

\section{Source and Study Populations}

The source population comprised all patients aged 18 years or older who initiated 1 or more courses of chemotherapy for a single primary cancer from July 1, 2003, to June 30, 2012. For each patient in the source population, all unique observed courses of chemotherapy were identified, as were all cycles within each of these courses. Patients in the source population were stratified into subgroups based on primary cancer type, presence of metastatic disease, and chemotherapy regimen.

From the source population, patients with primary cancers who initiated treatment with chemotherapy regimens classified as "low" or "intermediate" or unclassified in terms of FN risk in guidelines for CSF use were selected for inclusion in the study population. ${ }^{1,6,16}$ Regimen-specific subgroups with small sample sizes $(<3500$ patients, 
Febrile Neutropenia With Non-High-Risk Regimens

the minimum number needed for adequately powered analyses $[\beta=0.80]$ ) were excluded from consideration. Only the first course of chemotherapy with an eligible (ie, low-risk, intermediate-risk, or unclassified) regimen was considered in analyses. A complete description of methods used to identify cancer type and the presence/absence of metastatic disease, and to characterize chemotherapy courses, cycles, and regimens, is provided in supplemental eAppendices 2-4.

\section{Study Outcomes}

FN was ascertained on a cycle-specific basis, from day 6 of the chemotherapy cycle (ie, beginning on the 6th day after administration of the first chemotherapy agent during that cycle) to the last day of the chemotherapy cycle. FN was identified by the setting of care (inpatient vs outpatient) in primary analyses, using the diagnosis codes for neutropenia (International Classification of Diseases, Ninth Revision, Clinical Modification [ICD-9-CM] code 288.0), infections (see supplemental eAppendix 5), and fever (ICD-9-CM 780.6); the presence of any of these codes (ie, those for neutropenia or infection or fever) - per the algorithms presented herein - was considered evidence of FN. ${ }^{22-25}$

Inpatient $\mathrm{FN}$ was identified based on acute care facility admissions with a principal or secondary diagnosis code for FN (as mentioned previously). Outpatient FN was ascertained based on encounters in an ambulatory setting with a diagnosis code for FN (as mentioned previously) and, on the same date, evidence of intravenous (Health Care Common Procedure Coding System [HCPCS] level II codes) or oral (National Drug Codes [NDCs]) antimicrobial therapy. Outpatient encounters that preceded or followed a hospitalization during the same cycle of chemotherapy were not considered as separate outpatient FN episodes. Alternative definitions for FN were used in sensitivity analyses, including using only intravenous antimicrobial therapy for outpatient $\mathrm{FN}$, using only the diagnosis code for neutropenia, and using only intravenous antimicrobial therapy for outpatient FN and the diagnosis code for neutropenia.

\section{Risk Factors for FN and Other Variables}

Risk factors for FN consisted of those listed in clinical practice guidelines and those reported to increase the risk of FN in published literature, including age of 65 years or older, presence of selected chronic comorbidities, underweight and malnutrition; proxies for poor performance status (use of hospice care, skilled nursing facility, hospital bed, supplemental oxygen, walking aid, or wheel chair), use of immunosuppressive drugs, and history of selected blood-related disorders, infection, surgery (recent), and radiation therapy., ${ }^{13,6-14,27-34}$ Age was assessed based on the first day of the first cycle of chemotherapy in the course. All other risk factors (except for recent surgery and radiation therapy) were assessed during the 12-month period before the first day of the chemotherapy course; recent surgery was assessed during the 30-day prechemotherapy period, and radiation therapy during the 6 -month prechemotherapy period.

Other variables ascertained included the use of CSF, filgrastim, pegfilgrastim, and sargramostim, as prophylaxis in the first cycle of chemotherapy, which was defined as receipt on or before cycle day 5 . A detailed description of methods used to define FN risk factors (including all chronic comorbidities and blood-related disorders) and CSF use are provided in supplemental eAppendix 6.

\section{Statistical Analyses}

The percentage of subjects in each cancer- and regimen-specific subgroup with each of the individual risk factors and 1 or more risk factors was calculated, as was the distribution of subjects by the number of risk factors (ie, $0,1,2,3, \geq 4$ ). Incidence proportions for FN during the chemotherapy course were calculated within cancer- and regimen-specific subgroups, for patients with 1 or more risk factors and patients without risk factors, respectively; the relative risk (RR) of $\mathrm{FN}$ (ie, for patients with $\geq 1$ vs 0 risk factors) and corresponding 95\% CIs were also estimated. FN risk and corresponding RRs also were estimated by the number of risk factors present (ie, for patients with $1,2,3$, or $\geq 4$ vs 0 risk factors) and the number of chronic comorbidities (ie, those that are included among the list of risk factors). Analyses were conducted separately when considering the primary and alternative definitions of $\mathrm{FN}$, only FN episodes requiring inpatient care, and only FN episodes occurring in the first cycle of chemotherapy.

\section{Results}

A total of 160,304 patients received myelosuppressive chemotherapy with a regimen not classified as high risk for FN and met all other 
Weycker et al

inclusion/exclusion criteria (including $\mathrm{n} \geq 3500$ ), and thus were included in analyses; patients were stratified into 17 cancer- and regimen-specific subgroups (see supplemental eTable 1). The most common risk factors across the 17 subgroups were age of 65 years or older $(11 \%-55 \%$ of subjects $)$, cardiovascular disease $(15 \%-54 \%)$, diabetes $(10 \%-39 \%)$, osteoarthritis $(5 \%-17 \%)$, thyroid disorder $(6 \%-14 \%)$, and recent history of anemia $(10 \%-66 \%)$, infection $(30 \%-57 \%)$, or surgery (3\%-37\%) (Table 2). Most subjects within each subgroup had 1 or more risk factor, ranging from
74\% (nonmetastatic breast cancer, AC/AC-T [doxorubicin, cyclophosphamide, \pm docetaxel]) to $98 \%$ (metastatic lung cancer, carboplatin + paclitaxel) (Table 3), and many had 2 or more risk factors (range, 41\%-89\%) (see supplemental eFigure 1). Use of CSF prophylaxis in cycle 1, on an overall basis, varied substantially across regimens, and ranged from $0.3 \%$ (nonmetastatic colorectal cancer, 5-FU) to 63\% (NHL, R-CHOP [rituximab, cyclophosphamide, doxorubicin, vincristine, prednisone] every 3 weeks/every 4 weeks).

Incidence of $\mathrm{FN}$, based on the broad definition,

\begin{tabular}{|c|c|c|c|c|c|c|c|c|}
\hline & \multirow{2}{*}{\multicolumn{3}{|c|}{ Nonmetastatic Breast Cancer (\%) }} & \multicolumn{3}{|c|}{ Colorectal Cancer (\%) } & \multirow{2}{*}{\multicolumn{2}{|c|}{$\begin{array}{c}\text { Lung Cancer (\%) } \\
\text { Nonmetastatic } \\
\end{array}$}} \\
\hline & & & & \multicolumn{2}{|c|}{ Nonmetastatic } & \multirow{2}{*}{$\begin{array}{l}\text { Metastatic } \\
\text { FOLFOX } \\
(n=5,626)\end{array}$} & & \\
\hline & $\begin{array}{l}\text { TC } \\
(n=26,266)\end{array}$ & $\begin{array}{l}\text { TCH } \\
(n=9,105)\end{array}$ & $\begin{array}{l}\text { AC/AC-T } \\
(n=15,522)\end{array}$ & $\begin{array}{l}\text { FOLFOX } \\
(n=16,130)\end{array}$ & $\begin{array}{l}5-\mathrm{FU} \\
(\mathrm{n}=8,427)\end{array}$ & & $\begin{array}{l}\text { CAR+PAC } \\
(n=14,010)\end{array}$ & $\begin{array}{l}\text { CIS+ETO } \\
(n=6,669)\end{array}$ \\
\hline Age $\geq 65 y$ & 15.1 & 11.5 & 11.4 & 23.6 & 25.5 & 22.3 & 51.9 & 30.7 \\
\hline \multicolumn{9}{|l|}{ Chronic comorbidities } \\
\hline Autoimmune disease & 1.1 & 0.8 & 1.2 & 1.5 & 1.4 & 1.2 & 0.7 & 0.9 \\
\hline Cardiovascular disease & 16.8 & 14.6 & 16.6 & 33.8 & 25.8 & 40.5 & 53.7 & 45.2 \\
\hline Diabetes & 11.8 & 10.2 & 11.1 & 18.9 & 17.2 & 17.3 & 19.2 & 15.5 \\
\hline HIVIAIDS & 0.1 & 0.0 & 0.1 & 0.1 & 0.4 & 0.2 & 0.2 & 0.1 \\
\hline Liver disease & 1.9 & 2.2 & 2.0 & 7.6 & 5.7 & 24.1 & 3.6 & 4.3 \\
\hline Lung disease & 6.9 & 5.7 & 6.1 & 8.3 & 7.1 & 9.1 & 50.9 & 44.9 \\
\hline Renal disease & 1.3 & 0.9 & 1.0 & 3.2 & 2.8 & 3.4 & 4.2 & 1.7 \\
\hline Osteoarthritis & 7.0 & 6.1 & 6.0 & 5.5 & 4.8 & 5.1 & 8.7 & 9.1 \\
\hline Peptic ulcer disease & 0.2 & 0.2 & 0.2 & 1.4 & 0.8 & 1.2 & 0.7 & 0.7 \\
\hline Rheumatoid disease & 1.0 & 1.0 & 1.1 & 0.6 & 0.7 & 0.6 & 1.8 & 1.3 \\
\hline Thyroid disorder & 13.0 & 11.9 & 10.6 & 7.6 & 6.1 & 7.4 & 6.7 & 7.6 \\
\hline \multicolumn{9}{|l|}{ Number of comorbidities } \\
\hline 0 & 56.7 & 60.6 & 58.9 & 42.3 & 51.3 & 32.4 & 19.7 & 25.0 \\
\hline 1 & 30.0 & 28.4 & 29.5 & 34.6 & 30.8 & 36.9 & 32.6 & 34.9 \\
\hline 2 & 10.2 & 8.5 & 9.2 & 17.2 & 13.1 & 21.1 & 30.5 & 27.4 \\
\hline 3 & 2.4 & 1.9 & 2.1 & 4.7 & 3.7 & 7.5 & 13.1 & 9.7 \\
\hline$\geq 4$ & 0.7 & 0.5 & 0.4 & 1.3 & 1.0 & 2.1 & 4.2 & 3.0 \\
\hline Underweight or malnourished & 0.2 & 0.1 & 0.1 & 2.9 & 1.5 & 5.1 & 1.2 & 1.0 \\
\hline \multicolumn{9}{|l|}{ Proxies for poor performance status } \\
\hline $\begin{array}{l}\text { Hospice care or skilled nursing } \\
\text { facility }\end{array}$ & 0.3 & 0.2 & 0.2 & 1.3 & 1.0 & 1.7 & 1.5 & 0.7 \\
\hline \multicolumn{9}{|l|}{ Physical function } \\
\hline Use of hospital bed & 0.1 & 0.1 & 0.1 & 0.2 & 0.2 & 0.6 & 0.3 & 0.1 \\
\hline Use of supplemental oxygen & 1.0 & 0.6 & 0.8 & 1.6 & 1.4 & 2.1 & 12.4 & 6.9 \\
\hline Use of walking aid & 0.2 & 0.2 & 0.2 & 0.5 & 0.5 & 1.1 & 0.6 & 0.5 \\
\hline Use of wheel chair & 0.2 & 0.1 & 0.2 & 0.4 & 0.4 & 0.4 & 0.7 & 0.4 \\
\hline Any of above & 1.4 & 0.9 & 1.1 & 2.5 & 2.3 & 3.9 & 13.3 & 7.6 \\
\hline Any of above & 1.6 & 1.0 & 1.2 & 3.6 & 3.1 & 5.4 & 14.3 & 8.1 \\
\hline Use of immunosuppressive drugs & 6.9 & 8.2 & 3.1 & 2.0 & 1.7 & 2.5 & 8.1 & 5.6 \\
\hline \multicolumn{9}{|l|}{ Prechemotherapy conditions/events } \\
\hline Anemia & 11.2 & 10.3 & 11.0 & 44.0 & 26.8 & 45.0 & 20.0 & 16.0 \\
\hline Neutropenia & 5.7 & 5.1 & 4.9 & 5.3 & 2.8 & 7.1 & 5.4 & 6.4 \\
\hline Infection & 32.0 & 30.5 & 30.1 & 42.1 & 29.9 & 46.4 & 53.0 & 53.5 \\
\hline $\begin{array}{l}\text { Hospitalized for neutropenia, } \\
\text { fever, infection }\end{array}$ & 3.1 & 3.3 & 3.1 & 21.4 & 9.4 & 25.6 & 21.1 & 18.3 \\
\hline Recent surgery & 20.0 & 21.0 & 22.3 & 16.6 & 5.7 & 22.1 & 3.7 & 3.4 \\
\hline Radiation therapy & 7.6 & 4.1 & 3.3 & 8.3 & 78.8 & 6.3 & 51.5 & 59.6 \\
\hline
\end{tabular}

Abbreviations: 5-FU, fluorouracil; AC/AC-T, doxorubicin + cyclophosphamide (once q3wk or q4wk) +/- subsequent docetaxel or paclitaxel; BOR, bortezomib; CAR, carboplatin; CIS, cisplatin; ETO, etoposide; FOLFOX, folinic acid + fluorouracil + oxaliplatin; GEM, gemcitabine; MM, multiple myeloma; PAC, paclitaxel; R-CHOP, cyclophosphamide + doxorubicin + vincristine + prednisone with rituximab (once q3wk or q4wk); R-COP, cyclophosphamide + vincristine + prednisone with rituximab; TC, docetaxel + cyclophosphamide; TCH, docetaxel + cyclophosphamide + trastuzumab. 
Febrile Neutropenia With Non-High-Risk Regimens

during the chemotherapy course among patients with risk factors ranged from $7.2 \%$ (nonmetastatic colorectal cancer, 5-FU) to $29.0 \%$ (NHL, R-CHOP every 3 weeks/every 4 weeks), and $\mathrm{FN}$ incidence was generally higher among patients with risk factors than among those without (RR, range, 1.1-2.2 [95\% CI, 0.8-1.3 and 1.5-3.0, respectively]) (Table 3). FN incidence increased in a graded and monotonic fashion with the number of risk factors present. For example, in patients with nonmetastatic breast cancer receiving TC (docetaxel + cyclophosphamide) - the largest cancer/regimen subgroup, of whom 56.6\% received first-cycle CSF prophylaxis-FN incidence among those without risk factors was $10.1 \%$, and increased to $11.6 \%(\mathrm{RR}, 1.1 ; 95 \% \mathrm{CI}, 1.0-1.3)$ with 1 risk factor, to $13.9 \%(\mathrm{RR}, 1.4 ; 95 \% \mathrm{CI}, 1.3-1.5)$ with 2 risk factors, to $15.7 \%(\mathrm{RR}, 1.6 ; 95 \% \mathrm{CI}, 1.4-1.7)$ with 3 risk factors, and to $22.7 \%$ (RR, 2.2; 95\% CI, 2.02.5) with 4 or more risk factors. Corresponding values of FN incidence and RRs by the number of chronic comorbidities were as follows: no comorbidities, 14.1\%; 1 comorbidity, $18.3 \%$ (RR, $1.3 ; 95 \% \mathrm{CI}$, $1.2-1.4$ ); 2 comorbidities, 20.8\% (RR, 1.5; 95\% CI, $1.4-1.6) ; 3$ comorbidities, 22.0\% (RR, 1.6; 95\% CI,

Table 2 Prevalence of Risk Factors in Study Population, by Cancer Type and Chemotherapy Regimen (cont.)

\begin{tabular}{|c|c|c|c|c|c|c|c|c|c|}
\hline & \multicolumn{2}{|c|}{ Lung Cancer (\%) } & \multirow[b]{2}{*}{ MM (\%) } & & & \multicolumn{2}{|c|}{ Ovarian Cancer (\%) } & \multicolumn{2}{|c|}{ Pancreatic Cancer (\%) } \\
\hline & $\begin{array}{l}\text { Nonmetastic } \\
\text { CAR+ETO } \\
(n=4,886)\end{array}$ & $\begin{array}{l}\text { Metastatic } \\
\text { CAR+PAC } \\
(n=7,307)\end{array}$ & & \multicolumn{2}{|c|}{ NHL (\%) } & $\begin{array}{c}\text { Nonmetastatic } \\
\text { CAR+PAC } \\
(n=5,587)\end{array}$ & $\begin{array}{l}\text { Metastatic } \\
\text { CAR+PAC } \\
(\mathrm{n}=6,295)\end{array}$ & $\begin{array}{l}\text { Nonmetastatic } \\
\text { GEM } \\
(n=6,697)\end{array}$ & $\begin{array}{l}\text { Metastatic } \\
\text { GEM } \\
(n=4,452)\end{array}$ \\
\hline \multicolumn{10}{|l|}{ Chronic comorbidities } \\
\hline Autoimmune disease & 0.8 & 1.0 & 1.4 & 1.6 & 1.2 & 1.2 & 0.8 & 1.1 & 1.4 \\
\hline Cardiovascular disease & 50.1 & 34.8 & 32.4 & 33.0 & 24.9 & 38.1 & 38.5 & 41.4 & 32.4 \\
\hline Diabetes & 23.8 & 21.3 & 17.9 & 17.9 & 11.4 & 12.9 & 39.2 & 37.1 & 17.9 \\
\hline Lung disease & 50.1 & 38.9 & 10.9 & 9.7 & 11.0 & 7.2 & 8.4 & 10.8 & 10.9 \\
\hline Renal disease & 6.3 & 28.7 & 5.3 & 5.5 & 2.2 & 3.4 & 4.7 & 4.7 & 5.3 \\
\hline Osteoarthritis & 10.3 & 16.7 & 9.7 & 9.7 & 6.3 & 8.4 & 9.4 & 10.2 & 9.7 \\
\hline Peptic ulcer disease & 0.8 & 1.1 & 2.6 & 2.0 & 0.4 & 0.8 & 3.3 & 3.6 & 2.4 \\
\hline Rheumatoid disease & 1.6 & 1.5 & 1.9 & 1.9 & 1.0 & 1.2 & 0.9 & 1.0 & 1.9 \\
\hline Thyroid disorder & 8.0 & 9.5 & 11.0 & 9.5 & 13.0 & 14.4 & 8.8 & 9.8 & 11.0 \\
\hline$\geq 4$ & 6.1 & 3.9 & 5.3 & 2.8 & 2.9 & 1.0 & 2.1 & 4.4 & 6.4 \\
\hline Underweight or malnourished & 1.2 & 1.9 & 1.8 & 2.0 & 1.0 & 1.7 & 5.3 & 6.8 & 6.1 \\
\hline \multicolumn{10}{|l|}{ Proxies for poor performance status } \\
\hline $\begin{array}{l}\text { Hospice care or skilled nursing } \\
\text { facility }\end{array}$ & 2.6 & 2.1 & 3.7 & 1.6 & 2.3 & 1.6 & 3.3 & 2.8 & 2.2 \\
\hline \multicolumn{10}{|l|}{ Physical function } \\
\hline Use of hospital bed & 0.3 & 1.0 & 1.5 & 0.3 & 0.3 & 0.2 & 1.0 & 0.7 & 0.9 \\
\hline Use of supplemental oxygen & 13.0 & 9.3 & 3.3 & 2.3 & 2.8 & 1.7 & 2.8 & 2.5 & 2.7 \\
\hline Use of walking aid & 0.8 & 1.6 & 1.4 & 0.7 & 0.8 & 0.7 & 1.4 & 1.0 & 1.1 \\
\hline Use of wheel chair & 0.9 & 1.5 & 2.0 & 0.4 & 0.8 & 0.4 & 0.4 & 0.6 & 0.7 \\
\hline Any of above & 14.0 & 11.8 & 6.7 & 3.4 & 4.1 & 2.7 & 5.0 & 4.3 & 4.8 \\
\hline Any of above & 15.7 & 13.4 & 9.4 & 4.7 & 5.6 & 4.0 & 7.7 & 6.7 & 6.7 \\
\hline Use of immunosuppressive drugs & 6.5 & 20.7 & 18.4 & 9.3 & 8.7 & 6.4 & 7.1 & 2.5 & 3.1 \\
\hline
\end{tabular}

Abbreviations: 5-FU, fluorouracil; AC/AC-T, doxorubicin + cyclophosphamide (once q3wk or q4wk) +/- subsequent docetaxel or paclitaxel; BOR, bortezomib; CAR, carboplatin; CIS, cisplatin; ETO, etoposide; FOLFOX, folinic acid + fluorouracil + oxaliplatin; GEM, gemcitabine; MM, multiple myeloma; PAC, paclitaxel; R-CHOP, cyclophosphamide + doxorubicin + vincristine + prednisone with rituximab (once q3wk or q4wk); R-COP, cyclophosphamide + vincristine + prednisone with rituximab; TC, docetaxel + cyclophosphamide; TCH, docetaxel + cyclophosphamide + trastuzumab. 
Weycker et al

Table 3 Incidence Proportions for FN, by Presence/Absence of $\geq 1$ Risk Factor

\begin{tabular}{|c|c|c|c|c|c|}
\hline & $\%$ of Patients & $\%$ of Patients & Incidence Pro & tions for FN & \\
\hline & $\begin{array}{l}\text { With } \geq 1 \\
\text { FN Risk Factor }\end{array}$ & $\begin{array}{l}\text { With Cycle } 1 \\
\text { CSF Prophylaxis }\end{array}$ & $\begin{array}{l}\geq 1 \text { FN Risk } \\
\text { Factor }\end{array}$ & $\begin{array}{l}\text { 0 FN Risk } \\
\text { Factors }\end{array}$ & $\begin{array}{l}\text { Relative Risk } \\
(95 \% \mathrm{Cl})\end{array}$ \\
\hline Breast cancer & & & & & \\
\hline Nonmetastatic & & & & & \\
\hline TC $(n=26,266)$ & 79.0 & 56.6 & 17.4 & 12.9 & $1.3(1.2,1.5)$ \\
\hline $\begin{array}{l}\text { AC/AC-T, q3wk/q4wk } \\
(n=15,522)\end{array}$ & 74.4 & 43.3 & 20.0 & 12.9 & $1.6(1.4,1.7)$ \\
\hline $\mathrm{TCH}(n=9,105)$ & 75.2 & 58.2 & 19.5 & 15.1 & $1.3(1.2,1.4)$ \\
\hline Colorectal cancer & & & & & \\
\hline Nonmetastatic & & & & & \\
\hline FOLFOX $(n=16,130)$ & 90.2 & 10.2 & 12.6 & 8.7 & $1.4(1.2,1.7)$ \\
\hline $5-\mathrm{FU}(\mathrm{n}=8,427)$ & 96.6 & 0.3 & 7.2 & 6.3 & $1.2(0.7,1.8)$ \\
\hline Metastatic & & & & & \\
\hline FOLFOX $(n=5,626)$ & 93.5 & 9.7 & 11.3 & 6.1 & $1.9(1.2,2.8)$ \\
\hline Lung cancer & & & & & \\
\hline Nonmetastatic & & & & & \\
\hline CAR + PAC $(n=14,010)$ & 97.3 & 12.9 & 18.0 & 11.3 & $1.6(1.2,2.1)$ \\
\hline CIS + ETO $(n=6,669)$ & 96.6 & 18.1 & 23.2 & 14.9 & $1.6(1.1,2.1)$ \\
\hline CAR + ETO $(n=4,886)$ & 94.9 & 39.4 & 24.1 & 15.5 & $1.6(1.2,2.1)$ \\
\hline Metastatic & & & & & \\
\hline$C A R+P A C(n=7,307)$ & 97.9 & 18.3 & 20.4 & 10.6 & $1.9(1.2,3.1)$ \\
\hline Multiple myeloma & & & & & \\
\hline BOR $(n=7,009)$ & 95.4 & 1.2 & 20.8 & 9.6 & $2.2(1.5,3.0)$ \\
\hline Non-Hodgkin's lymphoma & & & & & \\
\hline $\begin{array}{l}\text { R-CHOP, q3wk/q4wk } \\
(n=12,324)\end{array}$ & 87.5 & 62.7 & 29.0 & 17.5 & $1.7(1.5,1.9)$ \\
\hline R-COP $(n=3,992)$ & 86.5 & 29.6 & 17.4 & 9.3 & $1.9(1.4,2.5)$ \\
\hline Ovarian cancer & & & & & \\
\hline Nonmetastatic & & & & & \\
\hline $\begin{array}{l}\text { CAR + PAC }(n=5,587) \\
\text { Metastatic }\end{array}$ & 87.0 & 22.8 & 12.6 & 10.6 & $1.2(1.0,1.5)$ \\
\hline CAR + PAC $(n=6,295)$ & 93.7 & 23.2 & 17.3 & 10.4 & $1.7(1.2,2.2)$ \\
\hline $\begin{array}{l}\text { Pancreatic cancer } \\
\text { Nonmetastatic }\end{array}$ & & & & & \\
\hline GEM $(n=6,697)$ & 93.3 & 1.6 & 18.3 & 12.0 & $1.5(1.2,2.0)$ \\
\hline Metastatic & & & & & \\
\hline $\operatorname{GEM}(n=4,452)$ & 94.6 & 23.1 & 23.1 & 21.9 & $1.1(0.8,1.3)$ \\
\hline
\end{tabular}

Abbreviations: 5-FU, fluorouracil; AC/AC-T, doxorubicin + cyclophosphamide (once q3wk or q4wk) +/- subsequent docetaxel or paclitaxel; BOR, bortezomib; CAR, carboplatin; CIS, cisplatin; CSF, colony-stimulating factor; ETO, etoposide; FN, febrile neutropenia; FOLFOX, folinic acid + fluorouracil + oxaliplatin; GEM, gemcitabine; PAC, paclitaxel; R-CHOP, cyclophosphamide + doxorubicin + vincristine + prednisone with rituximab (once q3wk or q4wk); R-COP, cyclophosphamide + vincristine + prednisone with rituximab; TC, docetaxel + cyclophosphamide; TCH, docetaxel + cyclophosphamide + trastuzumab.

$1.3-1.8$ ); and 4 or more comorbidities, $40.1 \%$ (RR, 2.8; 95\% CI, 2.4-3.4) (see supplemental eAppendix 7). Results were generally comparable across the 17 cancer/regimen-specific subgroups, and results were similar when using the alternative definitions for FN, when only FN episodes requiring inpatient care were considered, and when only FN episodes occurring in the first chemotherapy cycle were considered.

\section{Discussion}

NCCN Guidelines ${ }^{6}$ recommend prophylactic CSF for patients who, based on their chemotherapy regimen and risk factors, are at high-risk of FN. This recommendation, however, is based largely on findings from clinical trials, which may not be reflective of the population experience in clinical practice, on an overall basis (ie, for a given regimen), or within subgroups defined therein based on patient risk factors. Understanding these risks in this population is important for making informed treatment decisions at the patient level, because many patients receiving low- or intermediate-risk regimens may be at high risk of FN based on their risk factors. ${ }^{1,16,17,20}$ Understanding these risks is also important for making informed policy decisions at the population level, especially in light of 
Febrile Neutropenia With Non-High-Risk Regimens

recent publications suggesting the widespread use of CSF prophylaxis, which is inconsistent with guideline recommendations. ${ }^{15,17,35,36}$

We therefore undertook one of the first comprehensive evaluations to estimate the prevalence of risk factors for $\mathrm{FN}$ among patients with cancer receiving chemotherapy regimens not classified as high risk for FN in US clinical practice, and the RR of FN for patients with (vs without) risk factors and by the number of risk factors. We found that most patients (74\%-98\% across regimens) had 1 or more risk factor for $\mathrm{FN}$ and that many $(42 \%-88 \%)$ had 2 or more risk factors. Most importantly, we also found that FN risk was higher among patients with (vs without) a risk factor, especially among those with multiple risk factors.

Among the 5 most common cancer/regimen combinations, FN risk was $30 \%$ to $70 \%$ higher among those with 1 or more risk factor (vs no risk factors), and absolute FN risk was close to or exceeded the $20 \%$ threshold for most of these subgroups, suggesting that many patients with risk factors receiving low/intermediate-risk regimens are appropriate candidates for CSF prophylaxis. Among patients with NHL receiving R-CHOP, for example, absolute FN risk exceeded 20\% for all patients with risk factors, increasing from $21.9 \%$ among those with a single risk factor to $36.9 \%$ among those with 4 or more risk factors. Taken collectively, these findings underscore the importance of evaluating patient risk factors, both the type and number, in addition to the chemotherapy regimen when projecting FN risk and deciding whether to administer CSF prophylaxis. Such a personalized approach to cancer care and personal FN risk assessment is necessary to ensure optimization of patient outcomes. We note that, although estimates of FN risk-based on our operational algorithm for identifying $\mathrm{FN}$ episodesmay be somewhat different from true risks of FN, we believe that the magnitude of any bias resulting from imperfect case ascertainment is similar across subgroups and, accordingly, that it should not adversely impact our estimates of RRs. ${ }^{23}$ We also note that study results (ie, estimates of relative risk) were largely robust when using the alternative definitions for FN.

A few additional potential biases and limitations vis-à-vis patient (treatment) selection and outcome measurement deserve mention. Compared with subjects enrolled in clinical trials of low/intermediate- risk regimens, patients receiving these regimens in clinical practice may have a worse underlying risk profile, which may yield higher estimates of FN risk. On the other hand, these patients may be more likely to receive CSF or antimicrobial prophylaxis in clinical practice, which would lower the risk; dose reductions, regimen changes, and other $\mathrm{FN}$-related actions during the chemotherapy course may also impact study results. FN risk was estimated for each chemotherapy regimen considering all patients in that subgroup, irrespective of receipt of CSF prophylaxis. For this reason, study results may not necessarily reflect the true underlying risk of FN for a given regimen (ie, in the absence of supportive care).

We thus reported use of CSF prophylaxis to put study results into context. Although FN risk can be evaluated among the subset of patients who did not receive CSF prophylaxis, given that this subgroup is undoubtedly systematically different in terms of their FN risk profile, such analyses would inherently suffer from selection bias. For this reason, we decided not to conduct analyses limiting attention to patients who did not receive CSF prophylaxis.

Because FN rarely occurs within the first 5 days of a cycle, and this period was used to identify prophylactic CSF use, the follow-up period for ascertainment of FN began on cycle day 6 . However, to the extent that some patients actually experienced FN during the first 5 days of the cycle ( $<1 \%$ of patients in exploratory analyses using our broad definition), FN risks may be somewhat underestimated.

Because the accuracy of algorithms/variables capturing the presence of acute and chronic conditions is less than perfect, and because histories are left-truncated, some patients may be misclassified in terms of their risk factor profile.

Although not validated within the context of this study, measures of disability (ie, use of hospice care, skilled nursing facility, hospital bed, supplemental oxygen, walking aid, and wheel chair) have been found in previous evaluations to be predictive of performance status. ${ }^{29,31}$

Finally, because the study population comprised (principally) patients with cancer who were younger than 65 years with coverage from private US health plans, the study population may not reflect patients treated in clinical practice across the United States. 
Weycker et al

\section{Conclusions}

The results of this study suggest that most patients with cancer receiving chemotherapy regimens who are not classified as high risk for FN have 1 or more risk factors, and many have 2 or more. The results of this study also suggest that FN risk is elevated in these patients, especially those with multiple risk factors. For these reasons, and because patients with risk factors may also be at risk for more severe consequences of $\mathrm{FN}$, careful consideration should be given to fully evaluating each patient's overall risk of FN, based on a combination of the chemotherapy regimen and risk factors, so that those at elevated risk may be appropriately targeted for preventive measures. ${ }^{3,34}$

\section{References}

1. Smith TJ, Khatcheressian J, Lyman GH, et al. 2006 update of recommendations for the use of white blood cell growth factors: an evidencebased clinical practice guideline. J Clin Oncol 2006;24:3187-3205.

2. Lyman GH, Michels SL, Reynolds MW, et al. Risk of mortality in patients with cancer who experience febrile neutropenia. Cancer 2010;116:55555563.

3. Kuderer NM, Dale DC, Crawford J, et al. Mortality, morbidity, and cost associated with febrile neutropenia in adult cancer patients. Cancer 2006;106:2258-2266

4. Bonadonna G, Moliterni A, Zambetti M, et al. 30 years' follow up of randomized studies of adjuvant CMF in operable breast cancer: cohort study. BMJ 2005;330:217.

5. Lyman GH, Dale DC, Crawford J. Incidence and predictors of low doseintensity in adjuvant breast cancer chemotherapy: a nationwide study of community practices. J Clin Oncol 2003;21:4524-4531.

6. Crawford J, Becker PS, Armitage J, et al. NCCN Clinical Practice Guidelines in Oncology: Myeloid Growth Factors. Version 2, 2014. Available at: NCCN.org. Accessed June 8, 2015.

7. Chao C, Page J, Yang S, et al. History of chronic comorbidity and risk of chemotherapy-induced febrile neutropenia in cancer patients not receiving G-CSF prophylaxis. Ann Oncol 2014:25:1821-1829.

8. Chao C, Rodriguez R, Page J, et al. History of chronic comorbidity and risk of chemotherapy-induced febrile neutropenia in patients with non-Hodgkin lymphoma not receiving granulocyte colony-stimulating factor prophylaxis. Leuk Lymphoma 2015;56:72-79.

9. Lyman G, Abella E, Pettengell R. Risk factors for febrile neutropenia among patients with cancer receiving chemotherapy: a systematic review. Crit Rev Oncol Hematol 2014:90:190-199.

10. Chia V, Page J, Rodriguez R, et al. Chronic comorbid conditions associated with risk of febrile neutropenia in breast cancer patients treated with chemotherapy. Breast Cancer Res Treat 2013;138:621-631.

11. Hosmer W, Malin J, Wong M. Development and validation of a prediction model for the risk of developing febrile neutropenia in the first cycle of chemotherapy among elderly patients with breast, lung, colorectal, and prostate cancer. Support Care Cancer 2011;19:333-341.

12. Lyman G, Kuderer N, Crawford J, et al. Predicting individual risk of neutropenic complications in patients receiving cancer chemotherapy. Cancer 2011;117:1917-1927.

13. Lyman G, Lyman C, Agboola O. Risk models for predicting chemotherapyinduced neutropenia. Oncologist 2005;10:427-437.

14. Lyman G, Morrison V, Dale D, et al. Risk of febrile neutropenia among patients with intermediate-grade non-Hodgkin's lymphoma receiving CHOP chemotherapy. Leuk Lymphoma 2003;44:2069-2076.
15. Schnipper L, Smith T, Raghavan D, et al. American Society of Clinical Oncology identifies five key opportunities to improve care and reduce costs: the top five list for oncology. J Clin Oncol 2012;30:1715-1724.

16. Aapro MS, Bohlius J, Cameron DA, et al. 2010 update of EORTC 2011 guidelines for the use of granulocyte-colony stimulating factor to reduce the incidence of chemotherapy-induced febrile neutropenia in adult patients with lymphoproliferative disorders and solid tumours. Eur J Cancer 2011;47:8-32.

17. Kuderer N, Lyman G. Personalized medicine and cancer supportive care: appropriate use of colony-stimulating factor support of chemotherapy. J Nat Cancer Inst 2011;103:910-913.

18. Kuderer N, Culakova E, Poniewierski M, et al. Personalizing supportive care: a clinical prediction model for neutropenic complications in patient with early-stage breast cancer (ESBC) receiving intermediate risk cancer chemotherapy [abstract]. Cancer Res 2011;71:Abstract P5-20-03.

19. Lyman G, Culakova E, Poniewierski $M$, et al. Personalizing cance supportive care: predicting neutropenic complications in patients receiving intermediate risk cancer chemotherapy [abstract]. Blood 2011;118:Abstract 1022.

20. Crawford J, Armitage J, Balducci L, et al. Myeloid growth factors. J Natl Compr Canc Netw 2009;7:64-83.

21. Kuderer NM, Wolff AC. Enhancing therapeutic decision making when options abound: toxicities matter. J Clin Oncol 2014;32:1990-1993.

22. Weycker D, Li X, Edelsberg J, et al. Risk of febrile neutropenia in patients receiving emerging chemotherapy regimens. Support Care Cance 2014;22:3275-3285.

23. Weycker D, Sofrygin O, Seefeld K, et al. Technical evaluation of methods for identifying chemotherapy-induced febrile neutropenia in healthcare claims databases. BMC Health Serv Res 2013;13:60.

24. Weycker D, Edelsberg J, Kartashov A, et al. Risk and healthcare costs of chemotherapy-induced neutropenic complications in women with metastatic breast cancer. Chemotherapy 2012;58:8-18

25. Weycker D, Malin J, Edelsberg J, et al. Cost of neutropenic complications of chemotherapy. Ann Oncol 2008;19:454-460.

26. U.S. Department of Health \& Human Services. Code of Federal Regulations. Title 45: Public Welfare. Part 46: Protection of Human Subjects. Available at: http://www.hhs.gov/ohrp/humansubjects/guidance/45cfr46.html. Accessed July 4, 2013

27. Lyman GH, Kleiner JM. Summary and comparison of myeloid growth factor guidelines in patients receiving cancer chemotherapy. J Natl Compr Canc Netw 2007;5:217-228.

28. Crawford J. Risk assessment and guidelines for first-cycle colony-stimulating factor use in the management of chemotherapy-induced neutropenia Oncology (Williston Park) 2006;20(5 Suppl 4):22-28.

29. Davidoff A, Hurria A, Zuckerman I, et al. A novel approach to improve health status measurement in observational claims-based studies of cancer treatment and outcomes. J Geriatr Oncol 2013;4:157-165.

30. Davidoff A, Tang M, Seal B, Edelman MJ. Chemotherapy and survival benefit in elderly patients with advanced non-small-cell lung cancer. J Clin Oncol 2010;28:2191-2197.

31. Salloum R, Smith $T$, Jensen G, Lafata JE. Using claims-based measures to predict performance status score in lung cancer patients. Cance 2011;117:1038-1048

32. Lyman GH, Dale DC, Legg JC, et al. Assessing patients' risk of febrile neutropenia: is there a correlation between physician-assessed risk and model-predicted risk? [Published online ahead of print March 23, 2015. Cancer Med. doi: 10.1002/cam4.454

33. Culakova E, Poniewierski MS, Crawford J, et al. Impact of comorbidities on length of stay and mortality in hospitalized patients with cancer and febrile neutropenia [abstract]. Blood 2014;124:Abstract 2601.

34. Dulisse B, Li X, Gayle JA, et al. A retrospective study of the clinical and economic burden during hospitalizations among cancer patients with febrile neutropenia. J Med Econ 2013;16:720-735.

35. Waters G, Corrigan P, Gatesman M, Smith T. Comparison of pegfilgrastim prescribing practice to national guidelines at a university hospital outpatien oncology clinic. J Oncol Pract 2013;9:203-206.

36. Potosky A, Malin J, Kim B, et al. Use of colony-stimulating factors with chemotherapy: opportunities for cost savings and improved outcomes. J Nat Cancer Inst 2011;103:979-982. 